\section{Associative intrusions in short-term recall: A replication of Wickelgren '}

THOMAS J. HEFELE and JOSEPH LIEBERGALL, State University of New York at Buffalo, Buffalo, N.Y. 14214

Thirty-two undergraduates listened to lists of nine letters presented at the rate of four letters per second and then attempted to recall the letters in the correct order. Letters following repeated letters tended to be interchanged in recall. Such associative intrusions were observed whether the repeated letters occurred at the beginning or middle of the lists, whether they were separated by one or two letters, and whether or not the items following the repeated items had a common vowel phoneme. Except for a failure to confirm the backward intrusion phenomenon, the results agreed with the earlier findings of Wickelgren (1966) and added to the data base supporting Wickelgren (1969).

Wickelgren (1969) has recently examined problems in the interpretation of error data for STM and has concluded that it is not currently possible to decide whether this data can most appropriately be interpreted in auditory, articulatory, or abstract verbal terms. However, he does conclude that the phonetically coded STM trace is the principal one for recognition and recall of verbal items and of single vowel or consonant phonemes.

Conrad $(1959,1960)$ contributed to the empirical base leading to this conclusion. Conrad had demonstrated the tendency for position-to-item associations to occur in short-term serial learning. Subsequently, Wickelgren $(1965,1966)$ demonstrated the tendency for a second type of item association, viz, item-to-item associations, to occur. He determined that in lists such as "WYKDMDXHQ" the letters which follow the repeated letter (D) tend to be substituted for one another. In the example just cited, " $M$ " would tend to be substituted for " $X$ " in recall and vice versa. This type of item-to-item association error was referred to as an "associative intrusion."

An associative theory of STM was propounded by Wickelgren (1966) as a parsimonious explanation for this associative intrusion phenomenon. This theory states that associations are strengthened between the internal representatives of temporally adjacent items and that an item has only one internal representative regardless of how many times it appears in a list. Although correct responses are made more frequently than

$* p .05 ; * * p .01 ; * * * p .001$ incorrect ones due to the cues provided by serial position and by items preceding the repeated item, associative intrusions frequently occur when the two associates of the repeated item compete during recall. Since there is no a priori reason for assuming that the order of recall is from left to right, the backward direction must also be considered; therefore, confusion of the items preceding repeated items (backward intrusions) are also regarded as associative intrusions. Wickelgren (1966) found a significant tendency for associative intrusions to occur in both the forward and backward directions.

Since this Wickelgren (1966) experiment was particularly important in providing a foundation for current theoretical developments (e.g., Wickelgren, 1969), it was felt by the present investigators that a replication of that study would be worthwhile.

In order to establish the generality of the associative intrusion phenomenon, the following experimental conditions must be taken into account: (a) position of the repeated item in the list (beginning or middle); (b) number of items separating the repeated items (one or two); (c) the phonemic similarity of the items following the repeated items (similar or not similar); and (d) presence or absence of backward intrusions. The associative theory

propounded by Wickelgren $(1965,1966)$ predicts associative intrusions under all of these conditions.

\section{SUBJECTS}

The Ss were 32 undergraduates at the State University of New York at Buffalo, fulfilling a course requirement.

PROCEDURE

According to the design of Wickelgren (1966), eight types of lists of nine letters were presented to each of the Ss. The Ss listened to each of a total of 120 lists at the rate of approximately four letters per second and were then given $15 \mathrm{sec}$ to attempt to recall the lists in the correct order by filling in the boxes provided on their answer sheets. Immediately after the recall period the next list was presented. Each list contained two $/ \bar{e} /$ letters (B,C,D,E,G,P,T,V,Z), two / $/ \mathrm{e} /$ letters $(F, L, M, N, S, X)$, two $/ \bar{a} /$ letters $(A, H, J, K)$, two $/ \overline{\mathrm{u}} /$ letters $(\mathrm{U}, \mathrm{Q}, \mathrm{W})$, and one $/ \overline{\mathrm{i}} /$ letter $(I, Y)$. The experiment was recorded on tape and lasted about $40 \mathrm{~min}$.

The original tape used by Wickelgren (1966) was re-recorded such that the four blocks of 30 lists, each block containing three examples of each of the eight list types and also six all-different lists (i.e., without repeated items), were combined to form four different block sequences. One block sequence (I-II-III-IV) was that used by Wickelgren (1966), while the other three (II-IV-I-III; III-I-IV-II; and IV-III-II-I) were devised by recombining the blocks of his original sequence such that a given block did not appear twice in the same order and did not follow any other block more than once. Each block sequence was presented to 8 of

Table 1

Frequency of Associative vs Nonassociative Intrusions

\begin{tabular}{|c|c|c|c|c|c|}
\hline List Type & $\begin{array}{c}\text { Associative } \\
\text { Intrusions (\%) }\end{array}$ & $\mathbf{N}$ & $\begin{array}{l}\text { Forward Intrusions } \\
\text { All-Different } \\
\text { (Control) Intrusions }\end{array}$ & $\mathbf{N}$ & Difference \\
\hline$(\mathrm{ij} \mathrm{i} \widetilde{j})_{B}$ & 19.1 & 115 & 18.8 & 48 & +.3 \\
\hline$\left(\mathrm{i} \mathrm{j} \mathrm{i} \mathrm{k)_{B }}\right.$ & 10.2 & 128 & 9.8 & 173 & +.4 \\
\hline$(\mathrm{ij} \mathrm{i} \tilde{\jmath})_{M}$ & 21.6 & 37 & 0.0 & 8 & $+21.6^{*}$ \\
\hline$(\mathrm{ij} \mathrm{i} \mathrm{k})_{M}$ & 21.1 & 38 & 18.0 & 50 & +3.1 \\
\hline$\left(\mathrm{ij} 1 \mathrm{i} \hat{\mathrm{j}}_{\mathrm{B}}\right.$ & 35.5 & 107 & 0.0 & 21 & $+35.5^{* * * *}$ \\
\hline$(\mathrm{ij} 1 \mathrm{i} \mathrm{k})_{\mathrm{B}}$ & 24.3 & 115 & 5.7 & 87 & $+18.6^{* * *}$ \\
\hline$\left(\mathbf{i} j \perp \mathbf{i} \tilde{j}_{M}\right.$ & 13.3 & 30 & 22.2 & 9 & -8.9 \\
\hline$(\mathrm{ij} / \mathrm{ik})_{M}$ & 13.3 & 15 & 9.1 & 44 & +4.2 \\
\hline $\begin{array}{l}\text { Sim } \\
\text { Diff } \\
\text { B Sim } \\
\text { B Diff } \\
\text { M Sim } \\
\text { M Diff } \\
1 \text { Sim } \\
1 \text { Diff } \\
2 \text { Sim } \\
2 \text { Diff }\end{array}$ & $\begin{array}{l}24.9 \\
17.2 \\
23.5 \\
16.9 \\
17.9 \\
18.9 \\
14.4 \\
12.7 \\
30.7 \\
23.1\end{array}$ & $\begin{array}{r}289 \\
296 \\
255 \\
243 \\
67 \\
53 \\
243 \\
166 \\
137 \\
130\end{array}$ & $\begin{array}{r}12.8 \\
9.9 \\
13.0 \\
8.5 \\
11.8 \\
13.8 \\
16.1 \\
11.7 \\
6.7 \\
6.9\end{array}$ & $\begin{array}{r}86 \\
354 \\
69 \\
260 \\
17 \\
94 \\
56 \\
223 \\
30 \\
131\end{array}$ & $\begin{array}{c}+12.1^{* * *} \\
+7.3^{*} \\
+10.5^{* * *} \\
+8.4^{* *} \\
+6.1^{*} \\
+5.1 \\
-1.7 \\
+1.0 \\
+24.0^{* * *} \\
+16.2^{* * *}\end{array}$ \\
\hline $\begin{array}{l}\text { M } 1 \\
\text { M } 2\end{array}$ & $\begin{array}{r}10.0 \\
5.3\end{array}$ & $\begin{array}{l}40 \\
19\end{array}$ & $\begin{array}{c}\text { Backward Intrusions } \\
11.1 \\
6.1\end{array}$ & $\begin{array}{l}36 \\
33\end{array}$ & $\begin{array}{r}-1.1 \\
-.8\end{array}$ \\
\hline
\end{tabular}




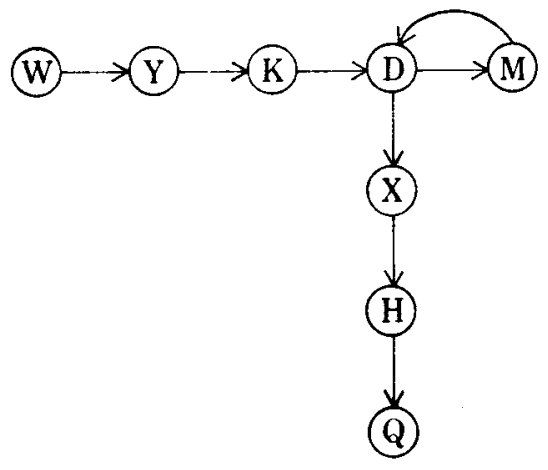

Fig. 1. Ilustration of asodiative theory of memory for WYKDMDXHQ.

the 32 Ss. This was done in order to eliminate any confounding due to a practice effect. The 120 trials presented in any one session followed one after another with no extra time between blocks, so that Ss were afforded no cues concerning the block structure of the experiment.

\section{RESULTS}

Associative intrusions were predicted only for the positions following or preceding correctly recalled repeated letters. Therefore, intrusions were counted only if the repeated letters were correctly recalled. The relative frequency with which these intrusions were associative, as opposed to nonassociative (any other correct letter), is shown in Table 1. For instance, $19.1 \%$ of the 115 intrusions that occurred in $(\mathrm{ijij})_{\mathrm{B}}$ after correctly recalled repeated letters were associative intrusions. In $19.1 \%$ of the cases, $j$ was substituted for $\hat{j}$ or $\hat{j}$ was substituted for j.

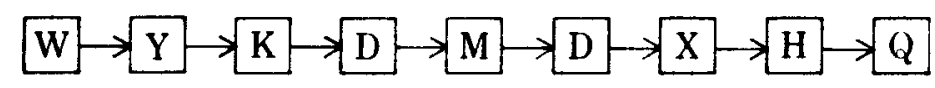

Fig. 2. Illustration of nonassociative theory of memory for WYKDMDXHQ.
To establish the associative phenomenon, it was necessary to determine if the relative frequency of associative intrusions was greater than would be expected merely on the basis of the close proximity of the two letters on the same list. This was done by comparing the frequency of associative intrusions to the frequency of confusing letters in the same position on the all-different lists. The control frequency for each type of list and each type of associative intrusion (forward and backward) was obtained by scoring the all-different lists in exactly the same manner as the list type containing the repeated letters. For instance, in lists where phonemically similar letters followed the repeated letters, only those all-different lists which contained phonemically similar letters in the corresponding positions were scored. The frequency of such intrusions in the control all-different lists (following correctly recalled subsequent or prior letters) is also shown in Table 1.

In the backward direction, no attempt was made to control systematically the similarity of the prior items and, therefore, cases where prior items were similar or different are lumped together. That is, "similar" and "different" are kept separate in the totals in Table 1 for intrusions in the forward direction but this was not done for the backward direction.

The relative frequency of associative and control intrusions were computed for each $S$, and the significance of the differences in relative frequency between associative and control intrusions were determined by the Wilcoxon matched-pairs signed-ranks test. One-tailed tests were used since the direction of the differences was definitely expected in advance to be positive.

In the forward direction, seven out of eight list types produced a greater relative frequency of associative than of control intrusions and three of these eight differences were significant. In the backward direction, neither list type (Ml or M2) produced a greater relative frequency of associative intrusions.

When conditions in Table 1 were combined, thereby increasing the number of intrusions, 9 out of the 10 resulting comparisons were in the expected direction and 7 of the 10 differences were statistically significant. For overall tests of significance, total sim and total diff were used; both comparisons were significant and total sim was significant beyond the .001 level. for our failure to replicate it for the backward direction, these results support the associative theory of short-term recall (Wickelgren, 1966), which holds that short-term associations are formed between adjacent items on a serial list. This theory is schematically represented in Fig. 1.

Each circle in Fig. 1 indicates the hypothetical internal representative of the item enclosed. The arrows between items indicate item-to-item associations in the forward direction. Note that although " $D$ " appears twice in the list it has only one internal representative. Therefore, the letters " $M$ " and " $X$," which are associates of the repeated letter, will be confused with a greater relative frequency than would be the case if they did not follow the repeated letter. Although the item " $D$ " has two associates (" $M$ " and " $X$ "), thus allowing for associative intrusions, correct recall should be more frequent since remote associations and serial position-to-item associations (not shown in Fig. 1) make the correct item the most likely association.

The associative theory of short-term recall can be contrasted with a nonassociative theory, as illustrated in Fig. 2. Such a theory assumes that items are stored in the temporal order of input, and that a repeated item can be stored in more than one cell. A nonassociative theory has difficulty in accounting for predominantly correct recall as well as for the fairly frequent manifestation of the associative intrusion phenomenon (Wickelgren, 1966).

In brief, the results of this replication, with the perplexing exception of the negative results with the backward intrusion phenomenon, contribute further to the empirical base for current theoretical developments in STM, particularly as spelled out by Wickelgren (1969). It remains for further research, however, to explain the difficulties encountered by the possible nonexistence of backward intrusions.

No consistent difference in the magnitude of the associative intrusion phenomenon was manifest over the different conditions. Furthermore, there was no practice effect on the relative frequency of associative and control intrusions. This was determined by comparing the difference in per cent for associative and control intrusions in both the total sim and total diff conditions for successive blocks of $\mathbf{3 0}$ lists. For total sim, the differences in per cent for successive blocks of 30 trials were $2.2,16.0,12.5$, and 15.2 ; and for total diff, the differences were 6.7,7.7,6.6, and 5.6.

\section{DISCUSSION}

The associative intrusion phenomenon which was demonstrated by Wickelgren (1966) has been replicated for the forward direction in the present experiment. Except

\section{REFERENCES}

CONRAD, R. Errors of immediate memory. British Joumal of Psychology, 1959, 50, 349-359.

CONRAD, R. Serial order intrusions in immediate memory. British Journal of Psychology, 1960, 51, 45-48.

WICKELGREN, w. A. Short-term memory for repeated and non-repeated items. Quarterly Journal of Experimental Psychology, 1965, 17, $14-25$.

WICKELGREN, W. A. Associative intrusions in short-term recall. Journal of Experimental Psychology, 1966, 72, 853-858.

WICKELGREN, W. A. Auditory or articulatory coding in verbal short-term memory. Psychological Review, 1969, 76, 232-235. NOTE

1. The authors are indebted to Dr. Wickeleren for the use of his original materials and his valuable suggestions. The authors are also grateful to Dr. Roy Lachman for his critical reading of the original manuscript. 